\title{
High Power Density Design of Power Electronic Interrupter in Hybrid DC Circuit Breaker
}

\author{
Jian Liu*, Lakshmi Ravi*, Dong Dong ${ }^{*}$, Rolando Burgos ${ }^{*}$, Cyril Buttay ${ }^{\dagger}$ and Steve Schmalz \\ Email: jianl@vt.edu \\ "Center for Power Electronics Systems, Virginia Tech, Blacksburg, VA, USA \\ †Univ Lyon, INSA-Lyon, CNRS, Laboratoire Ampère UMR 5005, F-69621, LYON, France \\ tEaton Corporation, Wisconsin, USA
}

\begin{abstract}
Circuit protection is a key enabler for future medium-voltage direct-current (MVDC) distribution systems. Hybrid dc circuit breaker (HCB) offers low conduction losses and reasonably fast response times, but suffers from large size. In this paper, a high power density power electronic interrupter design is introduced for the HCB. The device selection and tradeoff analysis of voltage clamping circuit are investigated. A small sized module with two parallel $1.7 \mathrm{kV}$ discrete IGBTs are selected as main switches. The RC snubber and MOV are carefully designed to guarantee no tail current bump and sufficient turn-off voltage margin. Experimental results at $12 \mathrm{kV}$ and $1 \mathrm{kA}$ are provided to verify the operation of the prototype.
\end{abstract}

Keywords-Hybrid dc circuit breaker, power electronic interrupter, high power density, trade-off analysis.

\section{INTRODUCTION}

DC power distribution system has been widely applied in dc Microgrid, data centers, electrical vehicle charging and multi-electric aircraft and so on [1]. For example, a future dc mircogrid is expected to integrate a range of ac source and clean energy including wind power, solar power, and battery storage. This kind of dc grid features lower cost, higher power flexibility, efficiency and quality [2].

Although medium-voltage dc (MVDC) system is very attractive, protection equipment of dc circuit breaker (DCCB) against short circuit fault represents a major technical barrier in development of MVDC networks [3]. Because the lack of natural zero crossing point and the faster rise of $\mathrm{dc}$ fault currents due to lower system inductance, bring several challenges to DCCB. DCCB can be categorized into three main types, mechanical circuit breaker (MCB), solid-state circuit breaker (SSCB) and hybrid circuit breaker (HCB). Compared to the other two type circuit breakers, HCB combines the low loss advantages of mechanical switches with the fast response times offered by solid-state switch [4]. Therefore, it is regarded as one of the most promising solutions.

A lot of $\mathrm{HCB}$ schemes have been proposed in the past, among which the HCB concept invented by ABB in 2012 (Fig. 1(a)) has achieved considerable success in the HVDC field. The unique advantage of the $\mathrm{ABB}$ solution is that the fault current can be forced to commutate from the mechanical branch to the electronic branch by turning off the load

This work was supported by the Advanced Research Projects AgencyEnergy (ARPA-E), under Award DE-AR0001111 in the BREAKER program monitored by Dr. Isik Kizilyalli.

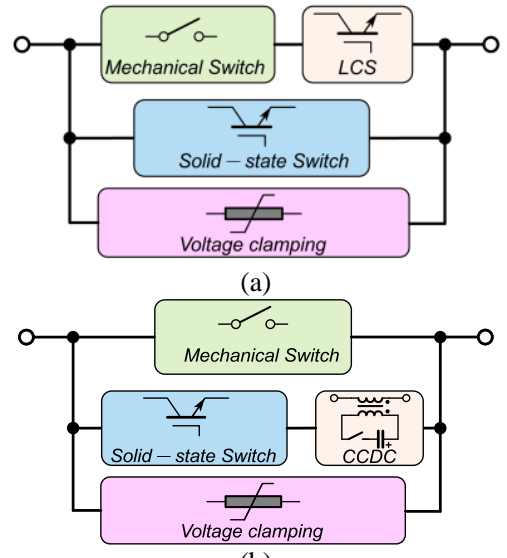

(b)

Fig. 1. Topologies of different kinds of HCB, (a) forced commutation HCB with LCS and (b) CCDC.

commutation switch (LCS), so it allows arcless opening of the mechanical contacts under a zero-current condition [5]. However, LCS introduces more conduction loss in the main branch and the cooling system may increase complexity and maintenance cost. Another solution of $\mathrm{HCB}$ with no conduction loss was proposed in [6], which adopts a current commutation drive circuit (CCDC) to force the current to commutate reliably and quickly (Fig. 1(b)). This structure has been used for the Zhangbei 500kV/25kA HCB, the world's largest capacity HVDC circuit breaker [7]. Currently, most HCB system is designed for the HVDC application, which focuses on the conduction loss, manufacturing cost, current interruption capability and maintenance convenience [8]. However, in the MV range, the power density is also an important factor to shrink the size and volume.

Considering the state of art bulky HCB system, this paper tries to increase the power density of current $\mathrm{HCB}$, especially focusing on the power electronic interrupter (PEI) part. In order to realize this target, the trade-off analysis and limiting factors of semiconductor devices are investigated in detail. Different voltage clamping circuits are compared to reduce the device stress, and a staged turn-off strategy is employed to lower the absorption energy as well as the breaking time. Finally, a medium voltage PEI prototype with breaking capability of 12 $\mathrm{kV}$ and $1 \mathrm{kA}$ are designed and built. The experimental results are given to verify the feasibility of proposed design. 


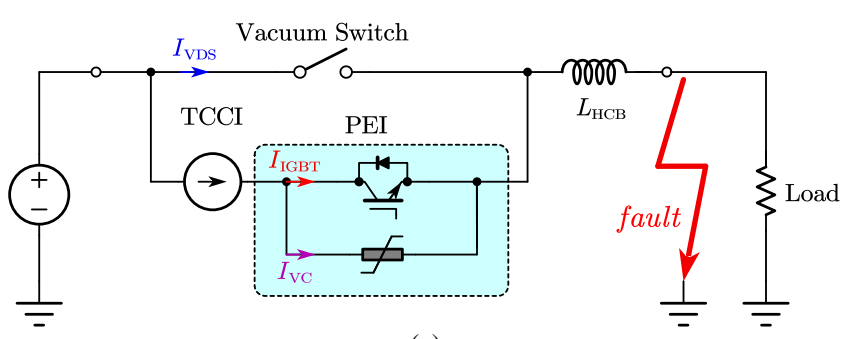

(a)

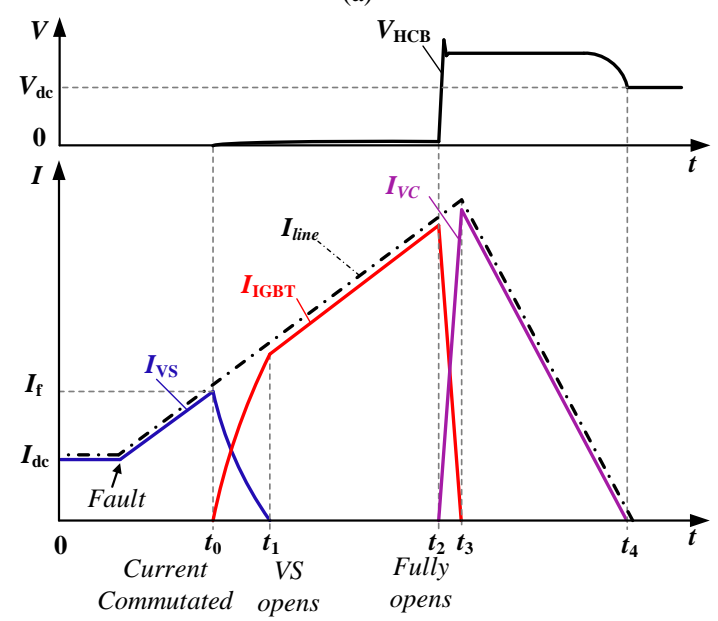

(b)

Fig. 2. (a) Structure of adopted HCB, (b) operation sequence and waveforms of HCB.

\section{High POWER DENSITY DESIGN OF PEI}

\section{A. HCB Structure and Modular PEI}

Fig. 2(a) shows the topology of the adopted HCB [9], which consists of three parts: the high speed vacuum switch (VS), the transient commutation current injector (TCCI) and PEI. The VS has favorable characteristics of arc extinguishing at the current zero point and uses the Thomson coil actuators to realize fast open of mechanical contactor [10]. The TCCI could be regarded as a controllable current source, which can track the main branch current and inject a current with opposite direction, so that VS could open at zero current condition. While the PEI consists of full-controlled solid-state paralleled voltage clamping circuit. Since insulated gate bipolar transistor (IGBT) has both high surge current ability, small size and easy series connection, thus it is selected as the best suitable device in this HCB application [11].

A simple turn-off sequence and waveform is shown in Fig. 2(b). At the normal state, the current flows through the closed VS, so the loss is very small. After short circuit happens and the fault is detected at $t_{0}$, the TCCI works to transfer the current from the VS to the PEI branch. The VS receives the trip command and generates enough gap between contactors after a certain time. When the current $I_{V S}$ reaches zero at $t_{1}$, the arc in VS is extinguished. Then the solid-state switch turns off at $t_{2}$, leaving the voltage clamping circuit to absorb the system energy and clear fault current. The fault clearing time is related to the line inductance and the ratio between $\mathrm{dc}$ bus and clamping voltage.

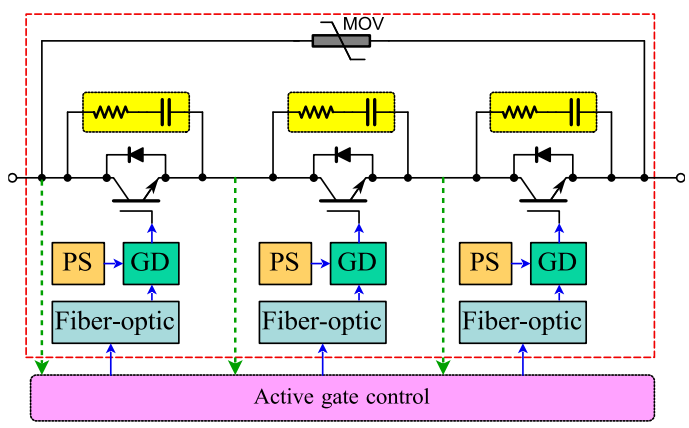

(a)

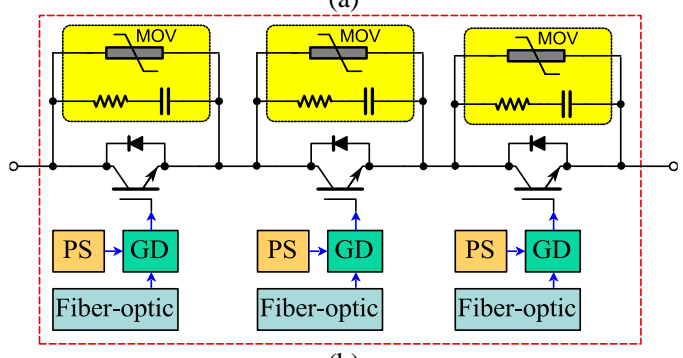

(b)

Fig. 3. (a) Convetional PEI structure uses a concentrated MOV, (b) modular PEI uses discrete MOV.

As an important part in $\mathrm{HCB}, \mathrm{PEI}$ is should interrupt $1 \mathrm{kA}$ peak current and withstand $12 \mathrm{kV}$ peak clamping voltage. Single device is hardly to meet the voltage requirement, which means series connected IGBT should be used here. Two types of PEI structure is shown in Fig. 3. A concentrated metal oxide varistor (MOV) is connected in parallel with the series IGBTs to limit the overvoltage and absorb energy. But additional voltage sharing technology such as passive snubber, active gate control and active clamping circuits should be utilized. Considering the MOV has the function of voltage clamping, a solution based on discrete MOV is proposed in [12] as shown in Fig. 1(b). In this case the RC snubber is used to lower switching losses and suppress the voltage spike of MOV. Compared to the former one, this method has better modularity and scalability, and can avoid cascading damage. Therefore, the modular PEI is selected here.

\section{B. PEI Device Selection}

Different from the normal PWM converter, which cares more about the nominal current, durations of the surge current events are very short due to the ultra-fast contact opening of the VS. Therefore, the requirements for IGBT in short-period high current operation are: 1) conduction of over-current without desaturation; 2) turn-off of peak current without latching (dynamic avalanche); and 3) the junction temperature $T_{\mathrm{j}, \max }$ should not be exceeded.

The power density and size are key points in this work, and the IGBT package plays an important role in the overall volume of power electronic interrupter. Therefore, several commercially available IGBT packages (both discrete and module) are considered. The current density is calculated by Eq. 1, and the comparison of all candidates is shown in Fig. 4.

$$
\begin{aligned}
& I_{\text {density }}=1000 /\left(A_{\text {device }} * N_{\text {parallel }}\right) \\
& N_{\text {parallel }}=1000 / I_{c m}
\end{aligned}
$$




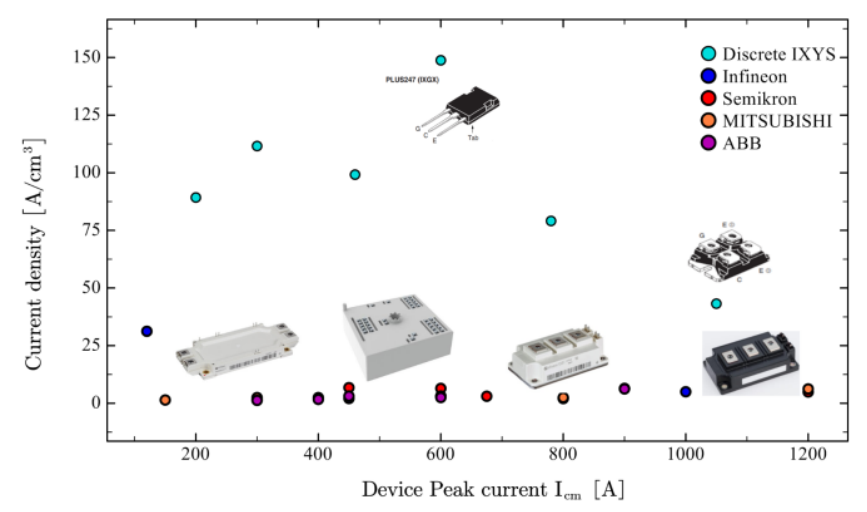

Fig. 4. Peak current density of comermical $1.7 \mathrm{kV}$ IGBT.

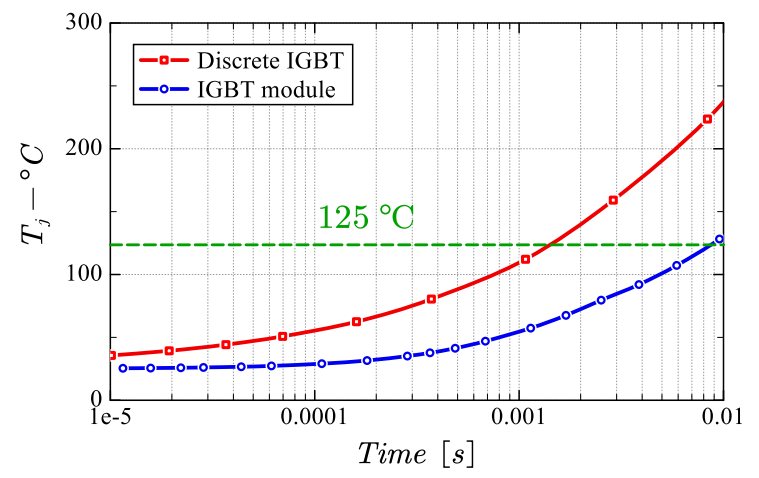

Fig. 5. Relationship between juction temperature $T_{\mathrm{j}}$ and $1 \mathrm{kA}$ pulse current duration.

Where $A_{\text {device }}$ is the package volume, $N_{\text {parallel }}$ is the parallel number and $I_{\mathrm{cm}}$ is the peak current from datasheet. It can be seen that the discrete $1.7 \mathrm{kV}$ IGBT has the highest current density which is essential to meet our power density goal. The discrete device also offers more flexibility in the design and is easier to parallel.

Apart from the peak current density, the current conduction ability is also compared between one discrete IGBT and one IGBT module. During the short pulse, the heat produced by junction will not reach the case, so the case temperature $T_{\mathrm{c}}$ could be considered same as the ambient temperature. In this way, the variance of $T_{\mathrm{j}}$ under $1 \mathrm{kA}$ peak current could be calculated through Eq. (2) and depicted in Fig. 5.

$$
T_{j}=T_{c}+\frac{P_{\operatorname{con}(1 k A)}}{Z_{t h}}
$$

Where $P_{\text {con }(1 \mathrm{kA})}$ is the device conduction loss with $1 \mathrm{kA}$ pulse current, and $Z_{\text {th }}$ is the device thermal impedance from junction to case. It should be noted that this variable is time related because of the existence of thermal capacitance. From Fig. 5 IGBT module has higher thermal capacitance to sustain peak currents for longer pulse duration $(>1 \mathrm{~ms})$. While discrete IGBTs can handle peak currents without thermal consequences for $<1 \mathrm{~ms}$. As the HCB acts within $500 \mu \mathrm{s}$ in our application, the discrete IGBT is sufficient. Since discrete IGBT has positive temp coefficient of $\mathrm{v}_{\mathrm{ce}(\mathrm{sat})}$, ideal for paralleling.

Above all, from the point of current density and current conduction ability, the discrete IGBT has much smaller size compared to the same voltage class IGBT module and there is

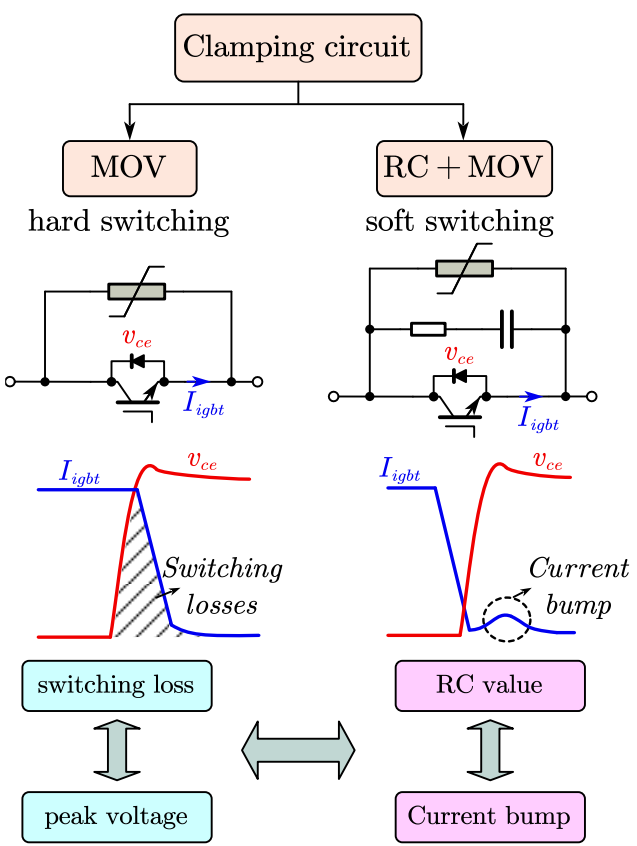

Fig. 6. Two types of voltage clamping circuit.

no any thermal problem during short pulse. Therefore, the discrete IGBT is more suitable for this application.

\section{Trade-off of Voltage Clamping Circuit}

As an important part of PEI, voltage clamping circuit works when IGBT is turned off. The fault current is commutated to this branch, and the energy is also absorbed by it. Generally, there are two types of voltage clamping circuit. One type is only MOV, another is MOV plus RC snubber. And when the fault current flows through the MOV, it can clamp the voltage to make sure it is smaller than device break over voltage. But it should be noted there is a voltage overshoot affected by the steep front effect and rapid rise of MOV current.

In the configuration of single MOV, the IGBT operates in a hard switching condition where it is exposed to simultaneous high current and high voltage, which can cause huge switching loss. The gate resistor is a freedom, when it is small, the turnoff speed is faster, so the switching loss is smaller but peak voltage is higher due to higher di/dt. Fig. 7 shows a test result of $3 \mathrm{kV} 90$ A IGBT. The IGBT could turn off 384 A current successfully, but fails when the current is increased to $432 \mathrm{~A}$. The switching loss increases from $395 \mathrm{~mJ}$ to $460 \mathrm{~mJ}$, which exceeds the thermal limit and leads to failure. Even if the device can survive this pulse, the performance and life-time may be degraded. Because of risk of device damage at turn-off from high peak current and clamping voltage close to the break down voltage, snubberless type single MOV voltage clamping circuit is not used here.

Another type is RC snubber paralleled with MOV, and the IGBT works in soft switching mode. The RC snubber serves two purposes: 1) helps lower the turn-off power/loss and 2) limits MOV voltage overshoot due to the steep front effect. However, there is a unique phenomenon of current bump when the current transfers from the IGBT to the snubber branch. This phenomenon has been reported in many papers about the soft- 


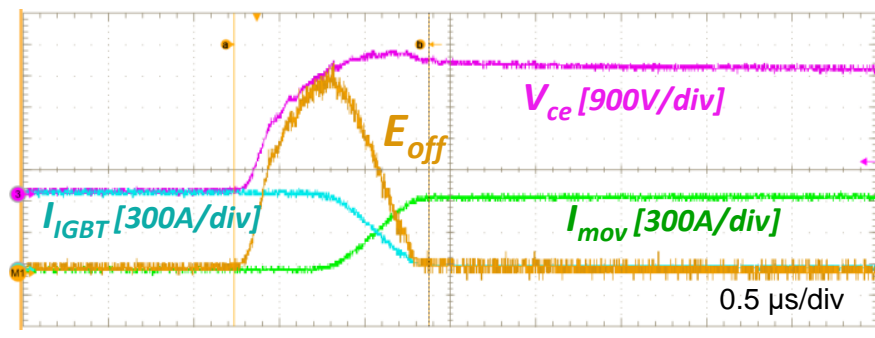

(a)

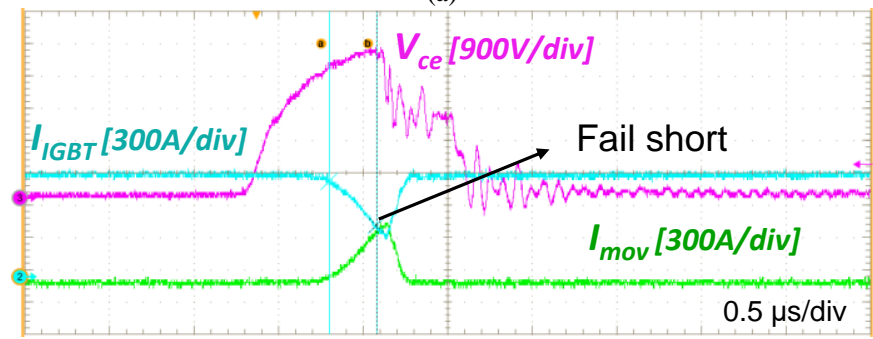

(b)

Fig. 7. Surge current turn-off waveforms for snubberless voltage clamping circuit: (a) successful turn-off of $384 \mathrm{~A}$ at $2000 \mathrm{~V} V_{p k}(395 \mathrm{~mJ}$ turn-off energy); (b) device failure at $432 \mathrm{~A}, 2140 \mathrm{~V} V_{p k}(460 \mathrm{~mJ}$ turn-off energy).

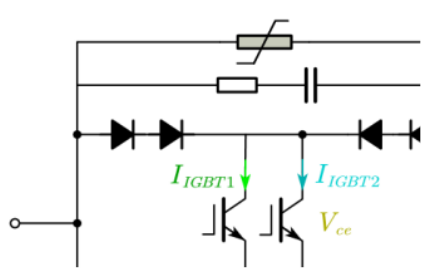

(a)

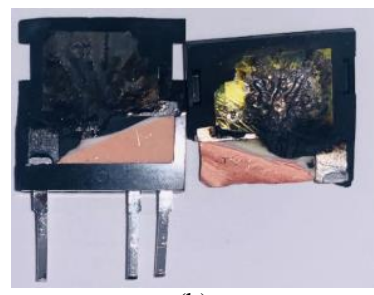

(b)
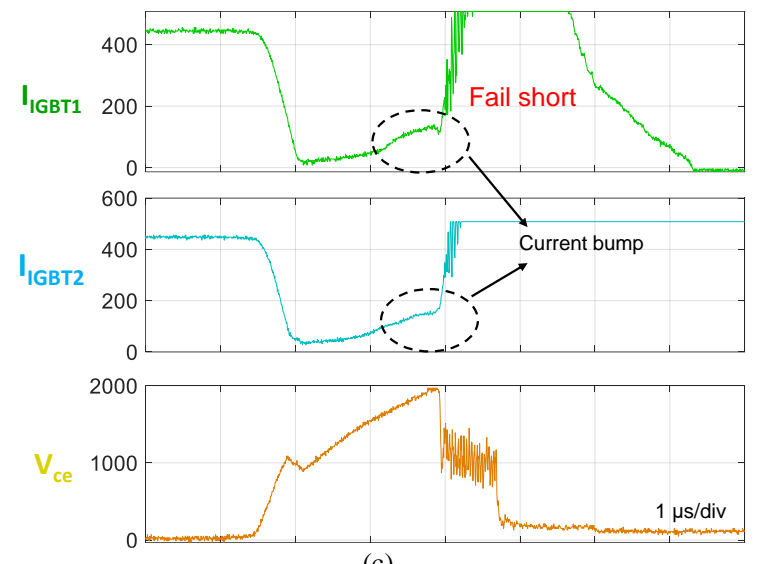

(c)

Fig. 8. (a) Circuit of two parallel IGBT with diode bridge and snuber type clamping circuit; (b) picture of damaged IGBT, (c) waveforms of IGBT failure at the current bump.

switching IGBT, which operates at nominal current [13]. In this case, the current bump only introduces some additional switching losses but doesn't cause device failure.

However, as shown in Fig. 8 IGBT was damaged at turnoff current of $450 \mathrm{~A}$ and clamping voltage of $2 \mathrm{kV}$. It can be seen at the peak of current bump, one IGBT fails short so that the voltage drops to zero. Compared to the normal PWM converter, our test has much higher peak current, so the current bump magnitude is higher. Higher "bump" magnitudes in the presence of high $d v / d t$ may lead to dynamic avalanche that can

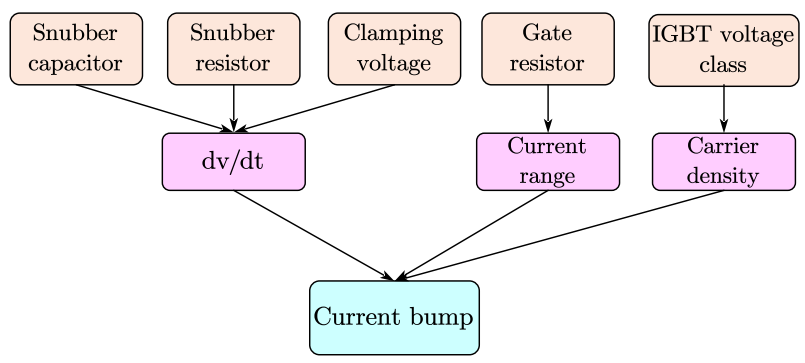

Fig. 9. Factors influencing tail current bump.

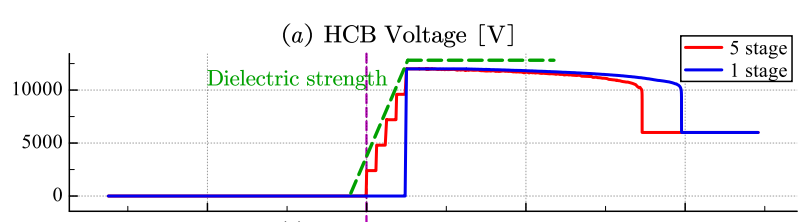

(b) Fault current [A]

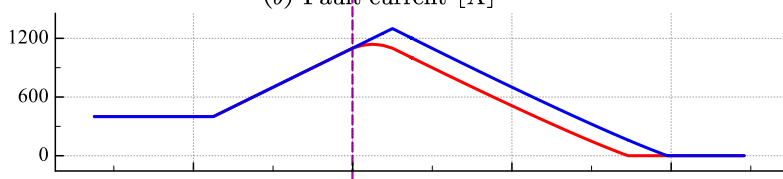

(c) Total MOV energy $[\mathrm{W}]$

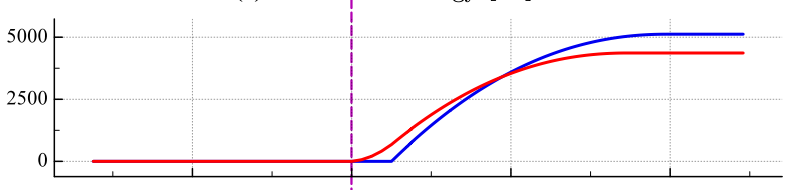

(d) Five modùle MOV energy $[J]$

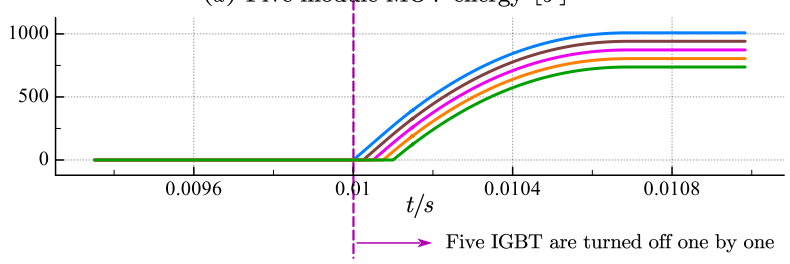

Fig. 10. Comparison between one stage and five stage turn-off sequence.

create filamentary currents. Such filamentation gives rise to a large local increase of temperature, causing device failure. The bump magnitude can be suppressed to acceptable levels using method shown in Fig. 9. After testing discrete IGBTs with different voltage classes, packages and techniques, the $1.7 \mathrm{kV}$ IGBT is selected. Because it does not need to lower the pulse current ratio or reduce the clamping voltage. According to the tuning result, $0.8 \mu \mathrm{F}$ capacitor and $1 \Omega$ resistor paralleled with MOV V25S510P are selected as voltage clamping circuit.

\section{Design of turn-off sequence}

The traditional HCB usually operates as shown in Fig. 2, PEI is allowed to be turned-off only after enough dielectric strength is established across contacts of VS. Otherwise, the arc will occur between contactor of VS and the current is commutated back to VS branch. However, this sequence requires a long waiting time for PEI.

Since the vacuum switch builds the dielectric strength gradually, the turn-off of PEI could start in advance. Once sufficient vacuum gap exists, the first IGBT could be turnedoff. Then the series IGBT could be turned-off one by one, only making sure the clamping voltage always smaller than the 


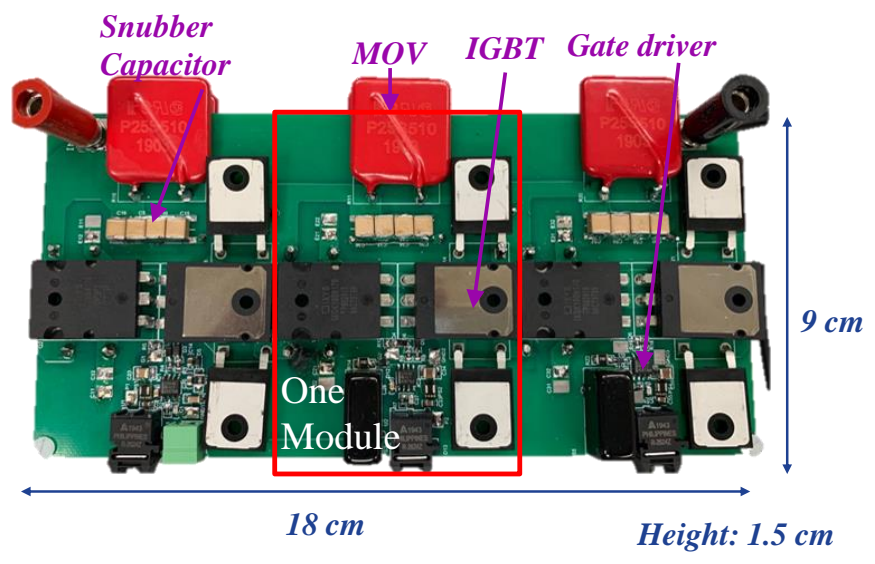

(a)

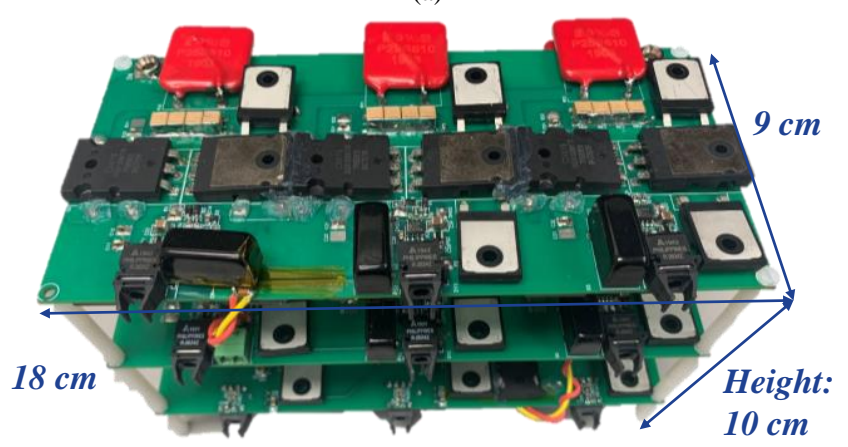

(b)

Fig. 11. (a) Single board with three series modules, (b) three stacked boards as $12 \mathrm{kV}$ PEI prototype.

dielectric strength. Take 5 stages as an example, the turning-off sequence is simulated in MATLAB, and the results are plotted in Fig. 10. It can be observed that the peak current reduces faster and whole fault current clearing time is reduced, the MOV energy absorption is decreased as well.

\section{12 KV PEI PROTOTYPE AND EXPERIMENTAL RESULTS}

Based on previous analysis, two parallel $1.7 \mathrm{kV} / 170 \mathrm{~A}$ discrete IGBTs are selected as the main switches. Fig. 11 presents a PCB board with three series modules, and three stacked boards are connected in series to obtain $12 \mathrm{kV}$ clamping voltage. It can be seen the total size is similar to two $4.5 \mathrm{kV} / 500 \mathrm{~A}$ IGBT modules from Infineon (single one is $14 \times 13 \times 5 \mathrm{~cm}^{3}$ ). But it should be noted that six IGBT modules are required to build a $12 \mathrm{kV}$ bidirectional PEI.

Simultaneous experimental results at $12 \mathrm{kV}$ and $1 \mathrm{kA}$ are shown in Fig. 12 (a). It can be seen the fault current is interrupted successfully, the voltage ripple after current at zero is caused by the oscillation between snubber capacitor, DC bus capacitor bank and line inductor. An improved staged turn-off strategy test results is shown in Fig. 12 (b), nine IGBTs are turned off one by one to make sure the clamping is always smaller than the dielectric strength between vacuum switch contactors. In this way, the peak voltage, current, MOV energy and interruption time could be reduced.
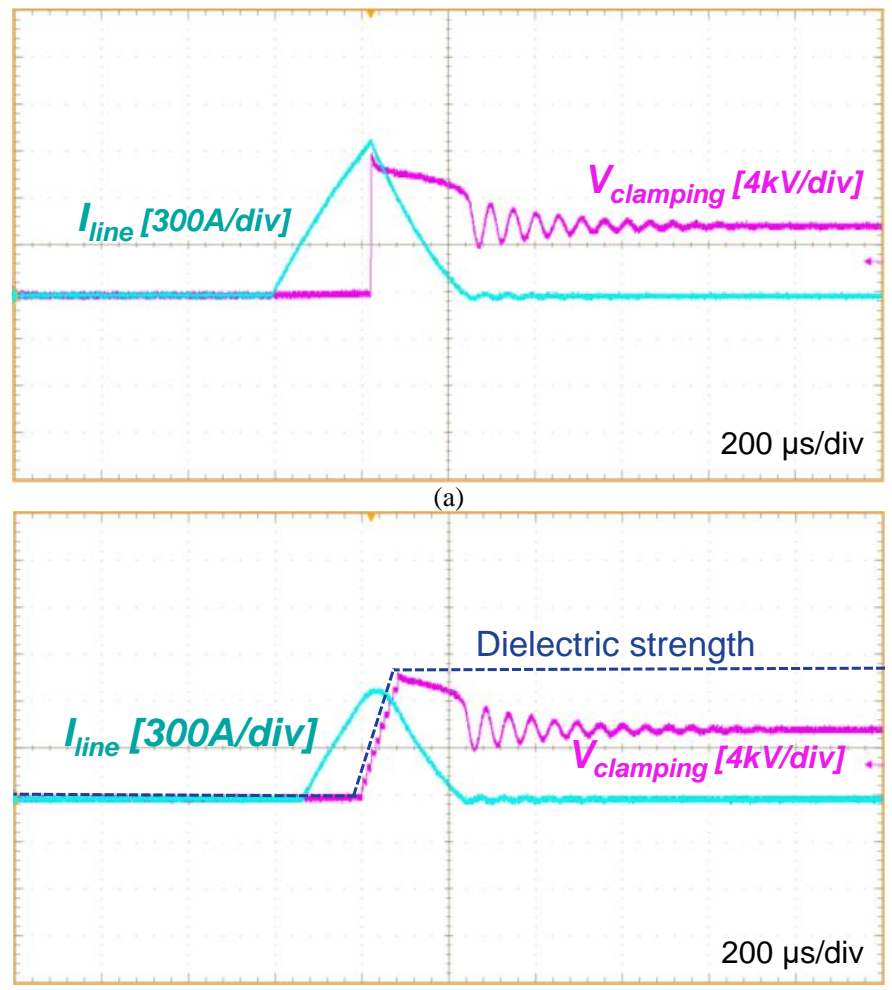

(b)

Fig. 12. Breaking waveform showing $12 \mathrm{kV}$ and $1 \mathrm{kA}$ peak PEI voltage and current, (a) simultaneous turn-off, and (b) staged turn-off.

\section{CONCLUSIONS}

In this paper, a high power density of modular PEI for medium voltage HCB. The modular design enables good scalability and voltage balance among series IGBTs. And inside each module, the discrete device is selected for $1 \mathrm{kA}$ pulse current to realize higher current density. RC plus MOV voltage clamping circuit is chosen, so that the current bump could be suppressed. The staged turn-off is employed to reduce the peak current and total energy. A full voltage $12 \mathrm{kV}$ PEI is also built to verify the function.

\section{ACKNOWLEDGMENT}

This work was supported by the Advanced Research Projects Agency-Energy (ARPA-E), under Award DEAR0001111 in the BREAKER program monitored by Dr. Isik Kizilyalli. The views and opinions of authors expressed herein do not necessarily state or reflect those of the United States Government or any agency thereof.

\section{REFERENCES}

[1] T. Dragičević, X. Lu, J. C. Vasquez and J. M. Guerrero, "DC Microgrids-Part I: A Review of Control Strategies and Stabilization Techniques," in IEEE Transactions on Power Electronics, vol. 31, no. 7, pp. 4876-4891, July 2016.

[2] A. Hooshyar and R. Iravani, "Microgrid Protection," in Proceedings of the IEEE, vol. 105, no. 7, pp. 1332-1353, July 2017. 
[3] S. Beheshtaein, R. M. Cuzner, M. Forouzesh, M. Savaghebi and J. M. Guerrero, "DC Microgrid Protection: A Comprehensive Review," in IEEE Journal of Emerging and Selected Topics in Power Electronics, to be published.

[4] R. Rodrigues, Y. Du, A. Antoniazzi and P. Cairoli, "A Review of SolidState Circuit Breakers," in IEEE Transactions on Power Electronics, vol. 36, no. 1, pp. 364-377, Jan. 2021.

[5] J. Hafner, "Proactive hybrid HVDC breakers-a key innovation for reliable HVDC grids," in Proc. CIGRE Bologna Symp., 2011, pp. 1-8.

[6] W. Wen, Y. Huang, Y. Sun, J. Wu, M. Al-Dweikat and W. Liu, "Research on Current Commutation Measures for Hybrid DC Circuit Breakers," in IEEE Transactions on Power Delivery, vol. 31, no. 4, pp. 1456-1463, Aug. 2016.

[7] X. Zhang et al., "A State-of-the-Art 500-kV Hybrid Circuit Breaker for a dc Grid: The World's Largest Capacity High-Voltage dc Circuit Breaker," in IEEE Industrial Electronics Magazine, vol. 14, no. 2, pp. 15-27, June 2020.

[8] Feng, Lei, et al. "Research on the breaking branch for a hybrid DC circuit breaker in $\pm 500 \mathrm{kV}$ voltage-sourced converter high-voltage direct current grid." IET Power Electronics 13.16 (2020): 3560-3570.

[9] Y. Zhou, Y. Feng, N. Shatalov, R. Na and Z. J. Shen, "An UltraEfficient DC Hybrid Circuit Breaker Architecture Based on Transient Commutation Current Injection," in IEEE Journal of Emerging and Selected Topics in Power Electronics, to be published.

[10] C. Peng, X. Song, A. Q. Huang and I. Husain, "A Medium-Voltage Hybrid DC Circuit Breaker-Part II: Ultrafast Mechanical Switch," in IEEE Journal of Emerging and Selected Topics in Power Electronics, vol. 5, no. 1, pp. 289-296, March 2017.

[11] Z. Chen et al., "Analysis and Experiments for IGBT, IEGT, and IGCT in Hybrid DC Circuit Breaker," in IEEE Transactions on Industrial Electronics, vol. 65, no. 4, pp. 2883-2892, April 2018.

[12] X. Zhang, Z. Yu, Z. Chen, Y. Huang, B. Zhao and R. Zeng, "Modular Design Methodology of DC Breaker Based on Discrete Metal Oxide Varistors With Series Power Electronic Devices for HVdc Application," in IEEE Transactions on Industrial Electronics, vol. 66, no. 10, pp. 7653-7662, Oct. 2019.

[13] S. Pendharkar and K. Shenai, "Zero voltage switching behavior of punchthrough and nonpunchthrough insulated gate bipolar transistors (IGBT's)," in IEEE Transactions on Electron Devices, vol. 45, no. 8, pp. 1826-1835, Aug. 1998. 